\title{
Memantine Treatment for Prevention of Neuronal Cell Death in Traumatic Brain Injury
}

\author{
R. Rosenthal ${ }^{1}$, C. Queenan ${ }^{1}$ and A. Waldron ${ }^{1}$ \\ 1. Bergen County Academies Nano-Structural Imaging Lab, 200 Hackensack Ave., Hackensack, NJ
}

Approximately 1.7 million traumatic brain injuries (TBIs) occur annually in the United States, but due to the complexity of the brain and cascade of events that constitute TBI, few pharmaceutical treatments have been developed [1]. One physiological effect of TBI is neuronal apoptosis caused by excitotoxicity, or cell death induced by excess neurotransmitter signaling, particularly by glutamate [2]. Injury-induced neurotransmitter release allows excess influx of $\mathrm{Ca}^{2+}$ ions that damage mitochondria and lead to neuronal cell death [3]. Prevention of excitotoxicity is an important step in developing a treatment for many types of TBI. Memantine hydrochloride is currently used to treat moderate Alzheimer's disease, with few side effects, and has potential for use in preventing excitotoxic cell death because it is a noncompetitive antagonist for the $\mathrm{N}$-methyl D-aspartate (NMDA) receptors activated by glutamate in vivo [4]. When the receptor is blocked, the excess $\mathrm{Ca}^{2+}$ responsible for cell death by excitotoxicty cannot enter the cell [5]. Additionally, the pharmacokinetics of memantine allow it to be used without significant detrimental effects to the normal function of neuronal signaling via glutamate [3].

In this study, two wells of a chamber slide were seeded with $1 \mathrm{~mL}$ human neuroblastoma (SH-SY5Y) cells in suspension and incubated until confluent. Once confluence was reached, both wells were uniformly scratched with a needle to simulate the physiological damage and inflammatory responses to TBI. Media in both wells was changed immediately following the scratch to remove debris. The control well was refilled with culture media, while the experimental well was refilled with $25 \mu \mathrm{M}$ memantine in culture media to simulate immediate treatment following TBI. The slide was then imaged by laser scanning confocal microscopy (LSCM) while maintained at $37^{\circ} \mathrm{C}$ and $5 \% \mathrm{CO}_{2}$. Cell migration was observed by recording still, brightfield images every 2 hours for 24 hours. Migration and proliferation were compared between control and Memantine treatment using ImageJ (Fig. 1). Images were converted to 8-bit and thresholding was used to distinguish area of cell coverage and empty area for analysis. Covered area was measured in each image and converted to percentage of original coverage (Fig. 2). The twelve points of control and experimental areas were plotted on a scatter plot and assigned a polynomial line of best fit to compare rates of healing. A student's t-test was applied to both sets of data. The progression of images was also compared qualitatively for completeness of healing and cell morphology.

Control and memantine treated cells showed very similar overall rates of growth. However, the wound in the post-treated memantine healed with less dense coverage than the control. This may be attributed to the different size scratches between the two samples, a fact that was accounted for in the quantitative data, but may have impaired intracellular communication in the treated sample and explained the somewhat sporadic growth. Alternatively, memantine could be seen as effective in promoting neuronlike regrowth due to the organic, connected nature of the regrowth of the treated cells, despite the smaller covered area. [6]

\section{References:}


[1] M Faul L Xu, M Wald, VG Coronado, "Traumatic Brain Injury in the United States: Emergency Department Visits, Hospitalizations and Deaths 2002-2006”. Atlanta (GA): Centers for Disease Control and Prevention, National Center for Injury Prevention and Control. 2010

[2] Margulies S, Hicks R et al. J Neurotrauma. 2009 June: 26 (6): 925-939.

[3] Meldrum BS. J. Nutr. 2000 April: 130(4): 1007-15.

[4] Hazardous Substances Data Bank. Bethesda (MD): United States National Library of Medicine. [date unknown].

[5] Volbracht C, van Beek J, Zhu C, et. al. Eur. J. Neurosci. 2006 23: 2611-2622.

[6] The authors would like to acknowledge the administration of the Bergen County Technical School district for their continued support of the research program.
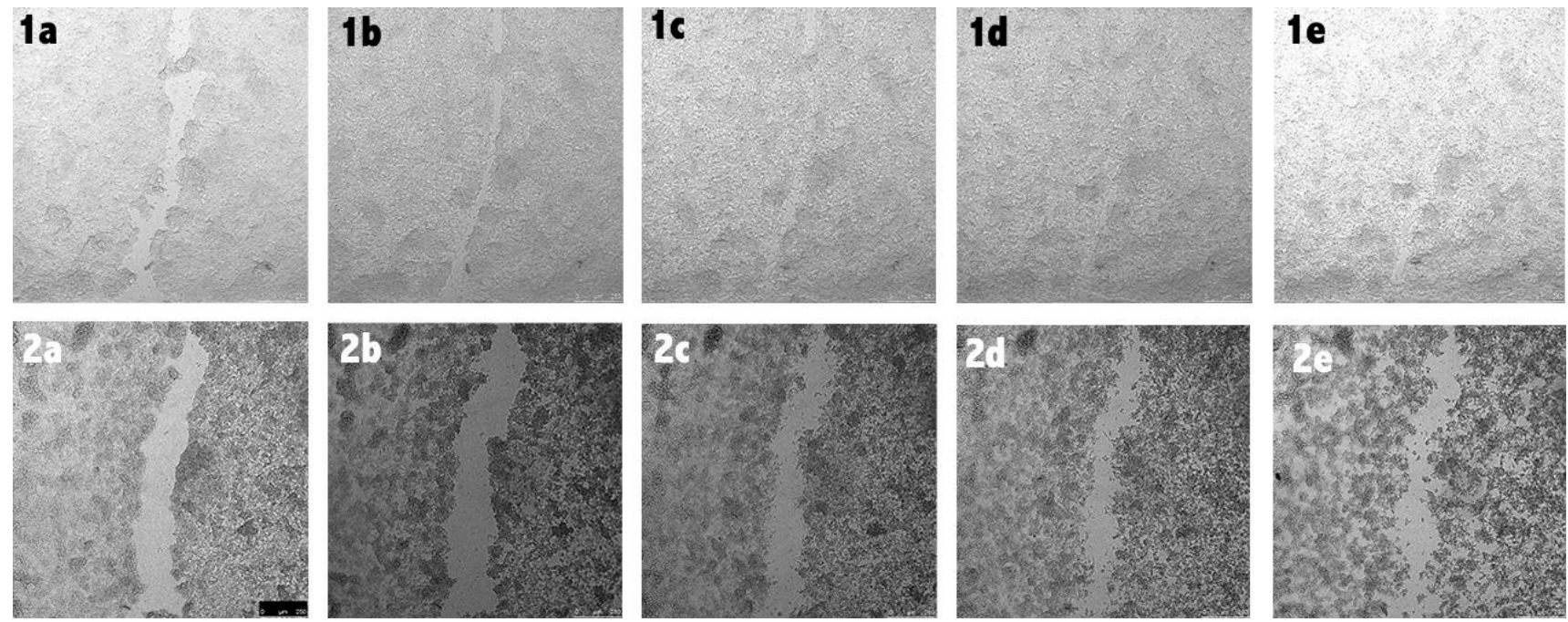

Figure 1. LSCM images of scratch assay performed on SH-SY5Y cells. 1) RPMI 1640 media control; 2) $25 \mu \mathrm{M}$ memantine at $0,6,12,18$ and 24 hours following injury (left to right). Images are $1.5 \mathrm{~mm}$ square.

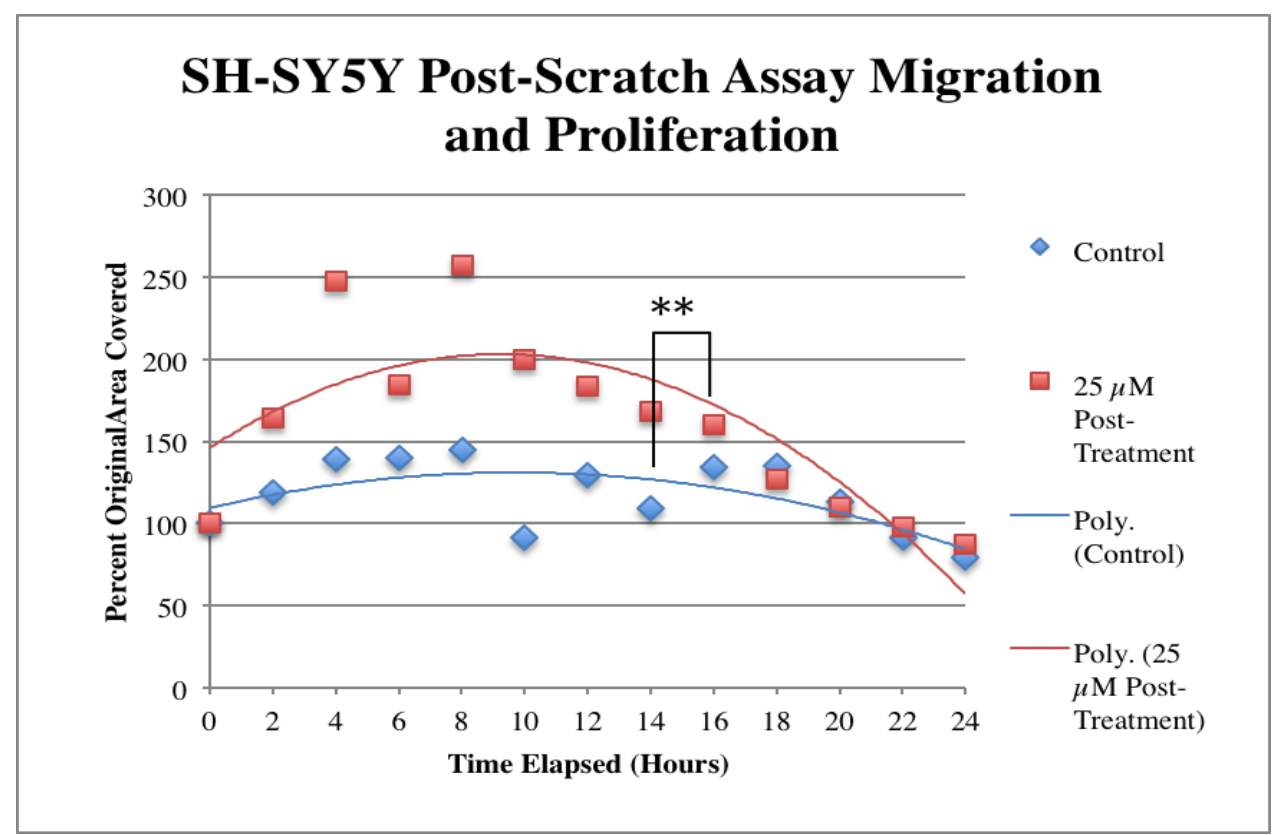

Figure 2. Analysis of area covered by control (blue) and treated (red) SH-SY5Y cells following scratch assay and converted to percent of original area $(\mathrm{t}=0)$. $* *$ indicates $\mathrm{p}<0.01$. 